\title{
The clinical utility of routine spinal radiographs by chiropractors: a rapid review of the literature
}

\author{
Melissa Corso ${ }^{1}$, Carol Cancelliere ${ }^{1}$, Silvano Mior ${ }^{1,2}$, Varsha Kumar $^{2}$, Ali Smith $^{2}$ and Pierre Côté ${ }^{*}$ (D)
}

\begin{abstract}
Introduction: When indicated by signs or symptoms of potentially serious underlying pathology (red flags), chiropractors can use radiographs to inform their diagnosis. In the absence of red flags, the clinical utility of routine or repeat radiographs to assess the structure and function of the spine is controversial.

Objectives: To determine the diagnostic and therapeutic utility of routine or repeat radiographs (in the absence of red flags) of the cervical, thoracic or lumbar spine for the functional or structural evaluation of the spine. Investigate whether functional or structural findings on repeat radiographs are valid markers of clinically meaningful outcomes. The research objectives required that we determine the validity, diagnostic accuracy and reliability of radiographs for the structural and functional evaluation of the spine.
\end{abstract}

Evidence review: We searched MEDLINE, CINAHL, and Index to Chiropractic Literature from inception to November 25, 2019. We used rapid review methodology recommended by the World Health Organization. Eligible studies (cross-sectional, case-control, cohort, randomized controlled trials, diagnostic and reliability) were critically appraised. Studies of acceptable quality were included in our synthesis. The lead author extracted data and a second reviewer independently validated the data extraction. We conducted a qualitative synthesis of the evidence.

Findings: We identified 959 citations, screened 176 full text articles and critically appraised 23 . No relevant studies assessed the clinical utility of routine or repeat radiographs (in the absence of red flags) of the cervical, thoracic or lumbar spine for the functional or structural evaluation of the spine. No studies investigated whether functional or structural findings on repeat radiographs are valid markers of clinically meaningful outcomes. Nine low risk of bias studies investigated the validity $(n=2)$ and reliability $(n=8)$ of routine or repeat radiographs. These studies provide no evidence of clinical utility.

Conclusion: We found no evidence that the use of routine or repeat radiographs to assess the function or structure of the spine, in the absence of red flags, improves clinical outcomes and benefits patients. Given the inherent risks of ionizing radiation, we recommend that chiropractors do not use radiographs for the routine and repeat evaluation of the structure and function of the spine.

Keywords: Spine, Radiograph, X-ray, Clinical utility, Chiropractor, Posture analysis

\footnotetext{
* Correspondence: pierre.cote@uoit.ca

${ }^{1}$ Faculty of Health Sciences, Ontario Tech University and Centre for Disability

Prevention and Rehabilitation, 2000 Simcoe St N, Oshawa, ON L1G 0C5,

Canada

Full list of author information is available at the end of the article
}

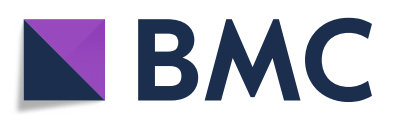

( ) The Author(s). 2020 Open Access This article is licensed under a Creative Commons Attribution 4.0 International License, which permits use, sharing, adaptation, distribution and reproduction in any medium or format, as long as you give appropriate credit to the original author(s) and the source, provide a link to the Creative Commons licence, and indicate if changes were made. The images or other third party material in this article are included in the article's Creative Commons licence, unless indicated otherwise in a credit line to the material. If material is not included in the article's Creative Commons licence and your intended use is not permitted by statutory regulation or exceeds the permitted use, you will need to obtain permission directly from the copyright holder. To view a copy of this licence, visit http://creativecommons.org/licenses/by/4.0/. The Creative Commons Public Domain Dedication waiver (http://creativecommons.org/publicdomain/zero/1.0/) applies to the data made available in this article, unless otherwise stated in a credit line to the data. 


\section{Introduction}

In the United States in 2010, the rate of spine radiographs within 5 days of presenting to a chiropractor was 204 per 1000 new patients [1]. An analysis of national trends in the United States suggests that the rate of spinal radiography by chiropractors and podiatrists increased by $14.4 \%$ between 2003 and 2015 [2]. This increase occurred despite the publication of several evidence-based clinical practice guidelines and clinical prediction rules to assist chiropractors in determining the indication for spine radiographs to assist with diagnosing a pathology [3-7]. Overall, guidelines suggest that radiographs are indicated when signs and symptoms of potentially serious underlying pathology (red flags) are identified through the clinical history and physical examination. However, on its own, an isolated "red flag" may have a high false positive rate for the diagnosis of underlying spinal pathology, such as cancer [8]. For example, the presence of a solitary "red flag" such as age over 50 years may not be sufficient to warrant taking spine radiographs [8, 9]. Therefore, clinicians are encouraged to combine sound clinical judgement and the assessment of red flags when ordering radiographs [9-11].

In the absence of "red flags", the use of spinal radiographs is not recommended [3-7]. Nevertheless, factions of chiropractors, including the International Chiropractic Association promote the use of routine or repeat radiographs to assess the structure and function of the spine [12-14]. This practice which dates back to 1910 was initiated when no evidence was available to guide the judicious use of spine radiographs [15]. Historically, these groups of chiropractors have argued that radiographs are helpful to measure postural abnormalities, identify vertebral misalignment or subluxation and guide treatment with spinal manipulative therapy $[12,15,16]$. The belief that radiographs are useful to detect and correct spine structure and function provides the foundation for many chiropractic technique systems that are still in use today. To our knowledge, approximately 23 chiropractic techniques use spine radiography (including full spine radiography) to guide the clinical management of patients [16]. These include the Gonstead, Chiropractic BioPhysics ${ }^{\bullet}$, Toggle-Recoil, and National Upper Cervical Chiropractic Association (NUCCA) techniques [16]. Proponents of these techniques claim that the use of routine and repeat radiographs is supported by scientific evidence and have published a guideline to assist clinicians with the biomechanical assessment of spinal subluxation in chiropractic clinical practice using radiography [13]. However, these claims have not yet been evaluated for their clinical utility, the benefit a patient gains from a test or treatment [17-19]. This was a particular concern for the College of Chiropractors of British Columbia (CCBC) which regulates the practice of chiropractic in the province of British Columbia, Canada. The mission of the $\mathrm{CCBC}$ is to protect the public by regulating British Columbia's doctors of chiropractic to ensure safe, qualified and ethical delivery of care [20].

At the request of the $\mathrm{CCBC}$, we conducted an independent rapid review of the literature to investigate the clinical utility of routine and repeat radiographs (in the absence of red flags) for the structural and functional evaluation of the spine by chiropractors. Specifically, we aimed to investigate: 1) the diagnostic utility of radiographs of the cervical, thoracic or lumbar region for the structural and functional evaluation of the spine; 2) the therapeutic utility of radiographs of the cervical, thoracic or lumbar region for the structural and functional evaluation of the spine; and 3) whether functional or structural findings on repeat radiographs of the cervical, thoracic or lumbar spine are valid markers of clinically meaningful change when monitoring conditions or managing patients. Our three main research objectives required that we first determine the validity and reliability of radiographs for the structural and functional evaluation of the spine.

\section{Methods}

We conducted a rapid review of the literature. Rapid reviews are used by health decision-makers (clinicians, patients, managers, and policy makers) who need timely access to health information to plan, develop and implement health care and policies [21, 22]. We used methodology recommended by the World Health Organization to answer our questions and previously used by our group $[21,23]$.

\section{Protocol and registration}

We reported our review according to the Preferred Reporting Items for Systematic Reviews and Meta-Analysis (PRISMA) and PRISMA Harms checklists [24, 25]. We registered our review with the International Prospective Register of Systematic Reviews (PROSPERO) on November 12, 2019 (CRD42020158321).

\section{Clinical utility}

Clinical utility is defined as the benefit that a person has from an intervention or test [17]. Clinical utility includes diagnostic utility (the degree to which the use of a test is associated with changing health outcomes) [18] and therapeutic utility (the degree to which a test contributes to improving health outcomes through the selection of an appropriate treatment) [19]. Demonstrating that a test has clinical 
utility requires demonstration that patients benefit from a test in a well-designed randomized clinical trial (RCT) or cohort study [17]. However, preliminary steps are necessary before the hypothesis that a clinical test has clinical utility can be tested (Fig. 1). First, the hypothesis that a test (e.g. spine radiographs) may benefit patient care must be generated from sound clinical observations. Second, the validity, diagnostic accuracy and reliability of the clinical test must be investigated [26-28]. Studies of diagnostic accuracy should report the sensitivity, specificity, predictive values and likelihood ratios of the test under investigation [26, 27] [Additional file 2]. Tests that are not valid, reliable or lack diagnostic accuracy are unlikely to have clinical utility, and therefore, unlikely to benefit patients [17-19]. Our methodology includes an evaluation of the diagnostic accuracy and reliability of radiographs used to evaluate the structure and function of the spine by chiropractors. Finally, tests that are reliable, valid and have diagnostic accuracy must demonstrate clinical (i.e., diagnostic and therapeutic) utility, in other words, impact health outcomes.

\section{Eligibility criteria}

\section{Participants and interventions}

We included studies of patients presenting to chiropractors who received spinal radiographs of the cervical, thoracic or lumbar region, in the absence of red flags.

\section{Comparators}

We considered comparisons with participants who did not receive spinal radiographs or were assessed with other spinal examination methods, such as palpation, postural evaluation or other diagnostic imaging techniques (such as CT scan or MRI).

\section{Outcomes}

We investigated structural or functional outcomes associated with various chiropractic approaches that use radiographs as diagnostic or assessment tools. Such approaches may include assessing for asymmetry in vertebral alignment as measured by line drawings, spinal curvatures, and the presence and correction of vertebral dysfunction as determined by measurement or positional listings. We also considered patient important outcomes throughout a course of treatment, including but not limited to pain, functioning, self-reported recovery, healthrelated quality of life, or well-being.

\section{Study designs}

We included RCTs, cohort studies, case-control studies, cross-sectional studies, and diagnostic and reliability studies. We excluded guidelines, letters, editorials, commentaries, unpublished manuscripts, dissertations, government reports, books and book chapters, conference proceedings, meeting abstracts, lectures and addresses, consensus development statements, guideline statements, cadaveric, laboratory or animal studies, qualitative studies, systematic reviews and meta-analyses.

\section{Information sources}

We developed our search strategy in consultation with a health sciences librarian, and a second librarian reviewed the strategy to ensure accuracy. We systematically searched three databases that thoroughly index the manual therapy literature published by various health professions from inception to November 25, 2019: MEDLINE (U.S. National Library of Medicine, through Ovid Technologies Inc.), Cumulative Index to Nursing and Allied Health (CINAHL, through EBSCOhost), and Index to Chiropractic Literature (ICL, Chiropractic Library Collaboration). Search terms consisted of subject headings specific to each database (e.g., MeSH in MEDLINE) and free text words relevant to our objectives and study design [see Additional file 1]. We restricted our search to papers published in English.

\section{Study selection}

We used a two-phase screening process to identify eligible studies. In phase one screening, we reviewed titles and abstracts and classified articles as possibly relevant or irrelevant. During phase two screening, we reviewed

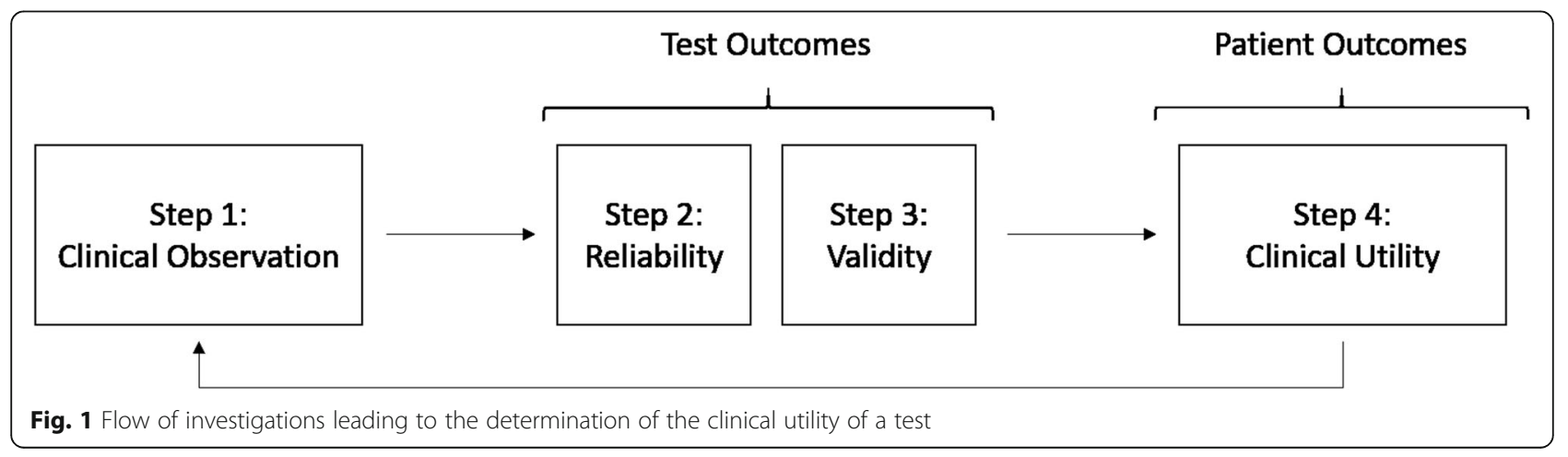


Table 1 Risk of Bias Tables

\begin{tabular}{|c|c|c|c|c|c|c|c|c|c|c|c|c|c|c|c|c|c|c|c|c|}
\hline \multicolumn{21}{|c|}{ Risk of Bias table: Diagnostic Studies } \\
\hline Author, Year & Phase & 1.1 & 1.2 & 1.3 & $R$ & C & 2.1 & 2.2 & $R$ & C & 3.1 & 3.2 & $\mathrm{R}$ & C & 4.1 & 4.2 & 4.3 & 4.4 & $R$ & Overall Ax \\
\hline Gregory, 1998 [34] & 1 & Y & $\mathrm{N} / \mathrm{A}$ & $\mathrm{N}$ & $\mathrm{H}$ & L & Y & N/A & $\mathrm{L}$ & L & CS & Y & $U$ & L & N/A & Y & Y & Y & L & At Risk of Bias \\
\hline Harrison, 2002 [35] & 1 & CS & N/A & Y & $U$ & L & CS & Y & $U$ & L & Y & CS & $U$ & L & N/A & Y & Y & Y & L & At Risk of Bias \\
\hline Frymoyer, 1986 [36] & 1 & Y & N/A & CS & $\mathrm{L}$ & L & Y & CS & $U$ & L & CS & Y & $U$ & L & N/A & Y & Y & Y & L & At Risk of Bias \\
\hline Harrison, 2003 [37] & 1 & CS & N/A & Y & $U$ & L & CS & Y & $U$ & $L$ & Y & CS & $U$ & L & N/A & Y & Y & Y & L & At Risk of Bias \\
\hline Wight, 1999 [38] & 1 & CS & N/A & Y & $U$ & L & CS & N/A & $U$ & L & Y & CS & $U$ & L & N/A & Y & Y & Y & L & At Risk of Bias \\
\hline Rosok, 1993 [39] & 1 & Y & N/A & CS & $L$ & L & $N$ & N/A & $U$ & L & Y & Y & L & L & N/A & Y & Y & CS & $L$ & At Risk of Bias \\
\hline Haas, 1992 [40] & 1 & CS & N/A & $\mathrm{N}$ & $\mathrm{H}$ & L & CS & N/A & $U$ & L & Y & Y & $\mathrm{L}$ & L & N/A & Y & Y & Y & $L$ & At Risk of Bias \\
\hline Haas, 1992 [41] & 1 & CS & N/A & $\mathrm{N}$ & $\mathrm{H}$ & L & CS & N/A & $U$ & L & Y & Y & L & L & N/A & Y & Y & Y & $L$ & At Risk of Bias \\
\hline Leboeuf, 1989 [42] & 1 & Y & N/A & Y & L & L & Y & Y & $L$ & $L$ & Y & CS & $U$ & L & N/A & Y & Y & Y & $L$ & At Risk of Bias \\
\hline Phillips, 1986 [43] & 1 & Y & N/A & CS & $\mathrm{L}$ & L & CS & N/A & $U$ & L & CS & Y & $U$ & $L$ & N/A & Y & Y & Y & $L$ & At Risk of Bias \\
\hline Rudy, 2015 [44] & 2 & $\mathrm{~N}$ & $\mathrm{~N} / \mathrm{A}$ & Y & $U$ & L & Y & Y & $L$ & $L$ & CS & Y & $\mathrm{H}$ & $L$ & N/A & Y & Y & Y & $L$ & At Risk of Bias \\
\hline McAviney, [45] & 2 & Y & Y & $\mathrm{N}$ & $L$ & L & Y & Y & $L$ & L & Y & Y & L & $L$ & N/A & Y & Y & Y & $L$ & Low Risk of Bias \\
\hline McGregor, 1995 [46] & 2 & Y & Y & CS & $L$ & L & Y & N/A & $\mathrm{L}$ & L & CS & Y & L & $\mathrm{L}$ & Y & Y & Y & Y & $\mathrm{L}$ & Low Risk of Bias \\
\hline
\end{tabular}

$Y$ yes; $N$ no; $C S$ Can't Say; $H$ high; $L$ low; N/A not applicable; $U$ Unclear; $R$ Risk; $C$ Concern

Legend: Diagnostic Studies, 1.1 Consecutive or Random Sample of Patients, 1.2 Case-control Design Avoided, 1.3 Avoid Inappropriate Exclusions, 2.1 Blinded Index Test Interpretation, 2.2 If Threshold Used, Pre-specified, 3.1 Reference Standard Classifies Condition, 3.2 Blinded Reference Test Interpretation, 4.1 Appropriate Interval Between Tests, 4.2 All Receive Reference Standard, 4.3 All Receive Same Reference Standard, 4.4 All Patients Included in Analysis

the full text of possibly relevant articles for final determination of eligibility.

A trained investigator (MC) conducted all of the screening. Prior to phase one and phase two screening, we validated the quality of screening by MC. Ten percent of all eligible articles were randomly selected and the titles and abstracts (phase one) and full text (phase two) of these articles were screened independently by a second experienced investigator (CC). A 95\% level of agreement was required between two reviewers before moving to full screening. Once the $95 \%$ agreement was achieved, one reviewer (MC) completed phase one and two screening.

\section{Risk of Bias in individual studies}

The lead author (MC) critically appraised the internal validity of relevant articles using the Scottish Intercollegiate Guidelines Network (SIGN) criteria for RCTs, cohort studies and case-control studies [29,30], a checklist created by Hoy et al. for cross-sectional studies [31], the Quality Assessment of Diagnostic Accuracy Studies (QUADAS) for diagnostic studies [32] and the Quality Appraisal tool for studies of diagnostic Reliability (QAREL) for reliability studies [33].

We included a quality control step in the critical appraisal of studies. The investigator who assessed the risk of bias of the studies (MC) presented a summary of the critically appraised papers to four experienced methodologists (PC, SM, CC, VK) who validated the outcome of the appraisals. Disagreements regarding the internal validity of papers were resolved through discussion. The lead author created risk of bias tables for all eligible studies (Tables 1 and 2), which were validated by the other investigators (PC, SM, CC, VK). Studies were rated low risk of bias or at risk of bias.

\section{Data extraction}

The lead author (MC) extracted data from acceptable quality (low risk of bias) studies and built evidence tables stratified by study type (Tables 3 and 4). Data extraction of each study was validated by one of four reviewers (PC, CC, SM, VK) to ensure accuracy. We contacted the study authors when clarification or additional information/data was necessary to build the evidence tables [46]. Evidence tables summarized the pertinent information and were used to create summary statements describing the body of evidence.

\section{Data items}

Information extracted from each diagnostic study included the study design, sample population, case definition, index test, reference standard and results of the study. Information extracted from each reliability study included study design, sample size, sample description, measurement method and results of the study.

\section{Statistical analyses}

When data were available, we computed the measurement mean change (and 95\% confidence intervals) from diagnostic studies. Confidence intervals (CI) were calculated using mean change in each group, standard 
Table 2 Risk of Bias Tables

\begin{tabular}{|c|c|c|c|c|c|c|c|c|c|c|c|c|c|}
\hline \multicolumn{14}{|c|}{ Risk of Bias table: Reliability Studies } \\
\hline Author, Year & 1.1 & 1.2 & 1.3 & 1.4 & 1.5 & 1.6 & 1.7 & 1.8 & 1.9 & 1.10 & 1.11 & 1.12 & Overall Assessment \\
\hline Assendelft, 1997 [47] & Y & Y & Y & U & U & N/A & Y & Y & Y & Y & Y & Y & Unacceptable (-) \\
\hline Frymoyer, 1986 [36] & Y & Y & Y & Y & $\mathrm{N} / \mathrm{A}$ & N/A & U & $u$ & U & U & Y & N & Unacceptable (-) \\
\hline Rosok, 1993 [39] & N & Y & U & N/A & Y & N & N/A & U & Y & U & Y & N & Unacceptable (-) \\
\hline Haas, $1992[40]^{a}$ & CS & CS & CS & CS & CS & CS & CS & CS & CS & CS & CS & CS & Unacceptable (-) \\
\hline Haas, $1992[41]^{a}$ & CS & CS & CS & CS & CS & CS & CS & CS & CS & CS & CS & CS & Unacceptable (-) \\
\hline Plaugher, 1990 [48] & $N$ & U & U & $U$ & U & N/A & Y & Y & U & U & Y & $N$ & Unacceptable (-) \\
\hline Phillips, 1986 [43] & Y & Y & Y & Y & N/A & N/A & U & U & Y & N/A & Y & N & $\begin{array}{l}\text { Unacceptable } \\
(-)\end{array}$ \\
\hline Janik, 2001 [49] & Y & Y & $U$ & U & $U$ & N/A & Y & Y & Y & Y & Y & Y & $\begin{array}{l}\text { Unacceptable } \\
(-)\end{array}$ \\
\hline Haas, 1990 [50] & Y & Y & Y & Y & N/A & N/A & Y & Y & U & N/A & $\begin{array}{l}\text { Rater 1 \& 2: } \\
\text { Y } \\
\text { Rater 3: } \\
\text { N }\end{array}$ & Y & $\begin{array}{l}\text { Rater } 1 \text { \& 2: Acceptable (+) } \\
\text { Rater 3: } \\
\text { Unacceptable (-) }\end{array}$ \\
\hline Troyanovich, 2000 [51] & Y & Y & Y & Y & Y & N/A & Y & Y & Y & $U$ & Y & Y & Acceptable (+) \\
\hline Troyanovich, 1998 [52] & Y & Y & Y & Y & Y & N/A & Y & Y & Y & U & Y & Y & Acceptable (+) \\
\hline Troyanovich, 1995 [53] & Y & Y & Y & Y & Y & N/A & Y & Y & Y & U & Y & Y & Acceptable (+) \\
\hline McGregor, 1995 [46] & Y & Y & Y & Y & Y & N/A & Y & Y & $N$ & Y & Y & Y & Acceptable (+) \\
\hline Harrison, $2002[54]^{b}$ & Y & Y & Y & Y & Y & N/A & Y & $u$ & Y & Y & Y & Y & Acceptable (+) \\
\hline Troyanovich, 1999 [55] & Y & Y & Y & U & U & N/A & U & Y & U & U & Y & Y & Acceptable (+) \\
\hline Jackson, 1993 [56] & Y & Y & Y & Y & Y & N/A & Y & Y & Y & Y & Y & Y & Acceptable (+) \\
\hline
\end{tabular}

$Y$ yes; $N$ no; $N / A$ not applicable; $U$ unclear; ++ high quality; + acceptable quality; - unacceptable quality/rejected

${ }^{a}$ No details about the methodology for the reliability study were reported in the paper

${ }^{b}$ Re-calculation on data from previous study, calculations reported in evidence table with original study

Legend: Reliability Studies, 1.1 Research Question, 1.2 Representative sample, 1.3 Representative raters, 1.4 Rater blinded to other raters, 1.5 Rater blinded to own findings, 1.6 Rater blinded to reference standard, 1.7 Rater blinded to clinical information, 1.8 Rater blinded to additional cues, 1.9 Order of examination, 1.10 Time interval between measurements, 1.11 Test application and interpretation, 1.12 Appropriate statistical measures

deviation, total number of participants in each group, and $\alpha=0.05$.

\section{Evidence synthesis}

We used the best evidence synthesis methodology to conduct a qualitative synthesis of the evidence from acceptable quality (low risk of bias) studies $[57,58]$. The evidence synthesis provides conclusions based on the best available evidence or may conclude that there is insufficient evidence to make any conclusions [57].

We stratified diagnostic studies into one of four phases, as described by Sackett [28]. Phase one studies test results in patients with the target condition compared to those without the target condition [28]. Phase two studies test whether patients with certain test results are more likely to have the target disorder than patients with differing results [28]. Phase three studies determine whether test results distinguish patients with and without the target disorder among patients in whom it is clinically reasonable to suspect the disease is present [28]. Phase four studies determine whether patients who undergo the test have improved health outcomes compared to similar patients who are not tested [28].

\section{Reporting of outcomes}

If we retrieved relevant RCTs, we aimed to check the clinical trials registry (Clinicaltrials.gov) to assess for outcome reporting bias.

\section{Results}

\section{Study selection}

Our search retrieved 1053 citations (Fig. 2). We removed 94 duplicates and screened 959 articles. Inter-rater agreement for phase one screening was $95.8 \%$ between MC and CC. We screened 176 full-text articles (phase two). Inter-rater agreement for phase two screening was 95.4\% between MC and CC. Of those, 23 articles met the inclusion criteria and were eligible for critical appraisal. Reasons for exclusion were ineligible publication type $(n=48)$, population not including patients presenting to chiropractors in the absence of red flags $(n=30)$, intervention did not include spinal radiographs $(n=12)$, did not have a comparison group $(n=27)$, outcomes 
Table 3 Evidence Tables

\begin{tabular}{|c|c|c|c|c|c|}
\hline \multicolumn{6}{|c|}{ Diagnostic Studies } \\
\hline $\begin{array}{l}\text { Author(s), } \\
\text { Year }\end{array}$ & $\begin{array}{l}\text { Design, } \\
\text { Sample size (n) }\end{array}$ & Case definition & Index test & Reference Standard & Validity \\
\hline $\begin{array}{l}\text { McAviney, } \\
2005 \text { [45] }\end{array}$ & $\begin{array}{l}\text { Study of criterion } \\
\text { validity } \\
\text { (Phase } 1 \text { for AWB } \\
\text { and Phase } 2 \text { for ARA) } \\
n=277\end{array}$ & $\begin{array}{l}\text { Cervical x-rays from randomly } \\
\text { selected patients from Summer } \\
\text { Hill Chiropractic Outpatient } \\
\text { Clinic (Macquarie University, } \\
\text { Australia), over } 7 \text { years } \\
\text { Exclusion: moderate to severe } \\
\text { degenerative changes; cervical } \\
\text { spine with obvious lordosis } \\
\text { and kyphosis; history of trauma. }\end{array}$ & $\begin{array}{l}\text { Sagittal cervical alignment } \\
\text { on x-ray films using posterior } \\
\text { tangent method: ARA of } \\
\text { cervical lordosis from C2-C7, } \\
\text { AWB of the head (horizontal } \\
\text { distance of posterior superior } \\
\text { body of C2 compared to } \\
\text { vertical line from posterior } \\
\text { inferior body of C7) } \\
\text { Partitioned into categories } \\
\text { with increments of } 5^{\circ}\end{array}$ & $\begin{array}{l}\text { Presence/Absence of } \\
\text { cervical complaints: } \\
\text { patients' records, history } \\
\text { in intern's radiology } \\
\text { report and x-ray referral } \\
\text { slip }\end{array}$ & $\begin{array}{l}\text { ARA: } \\
\text { Cervical complaint: } 9.6^{\circ} \\
\text { Non-cervical complaint: } \\
23.4^{\circ} \\
\text { ARA < } 20^{\circ} \text { (to identify } \\
\text { cervical complaint) } \\
\text { Sn: } 0.724 \\
\text { Sp: } 0.737 \\
\text { AUC: } 0.803 \\
\text { AWB: } \\
\text { Cervical complaint: } \\
21.3 \text { mm } \\
\text { Non-cervical complaint: } \\
21.1 \mathrm{~mm} \\
\text { NS difference between } \\
\text { groups }\end{array}$ \\
\hline $\begin{array}{l}\text { McGregor, } \\
1995 \text { [46] }\end{array}$ & $\begin{array}{l}\text { Phase } 2 \text { study } \\
n=512\end{array}$ & $\begin{array}{l}\text { New patients, > } 18 \text { YO, } \\
\text { Canadian Memorial } \\
\text { Chiropractic College } \\
\text { outpatient clinic, neck } \\
\text { and/or head pain, excluding } \\
\text { patients diagnosed with } \\
\text { pathology } \\
\text { Asymptomatic subjects from } \\
\text { small normative group from } \\
\text { a different study } \\
\text { Assessed for intersegmental } \\
\text { clinical hypermobility: mobility } \\
\text { of a given motion unit in the } \\
\text { cervical spine which is excessive } \\
\text { and is accompanied by local } \\
\text { and/or peripheral symptoms }\end{array}$ & $\begin{array}{l}\text { AP, lateral, AP open-mouth, } \\
\text { forward flexion and extension } \\
\text { cervical radiographs } \\
\text { Including history and } \\
\text { physical examination } \\
\text { findings summarized in a } \\
\text { standardized case report } \\
\text { form }\end{array}$ & $\begin{array}{l}\text { AP, lateral, AP open- } \\
\text { mouth cervical } \\
\text { radiographs } \\
\text { Including history and } \\
\text { physical examination } \\
\text { findings summarized } \\
\text { in a standardized } \\
\text { case report form }\end{array}$ & $\begin{array}{l}\text { With flexion-extension } \\
\text { radiographs ( } 3 \text { raters): } \\
\text { Sn: } 0.65-0.89 \\
\text { Sp: } 0.49-0.92 \\
\text { Without flexion-extension } \\
\text { radiographs ( } 3 \text { raters): } \\
\text { Sn: } 0.11-0.91 \\
\text { Sp: } 0.64-0.99\end{array}$ \\
\hline
\end{tabular}

$A P$ anteroposterior; $A R A$ absolute rotation angle; $A W B$ anterior weight bearing; $D D D$ degenerative disc disease; $D J D$ degenerative joint disease; $L R+$ positive likelihood ratio; $L R$ - negative likelihood ratio; NS no significant; $P P V$ positive predictive value; NPV negative predictive value; $R O C$ receiver operating characteristic; Sn Sensitivity; Sp Specificity; VAS visual analog scale

were not structural or functional findings on radiographs $(n=33)$ and duplicates $(n=3)$.

\section{Risk of Bias}

We found no relevant studies investigating the diagnostic or therapeutic utility of routine or repeat radiographs for structural or functional evaluation of the spine. Similarly, we found no studies investigating the use of repeat radiographs for functional or structural findings of the spine to monitor clinically meaningful changes in conditions or care for patients (Fig. 2).

We critically appraised 23 studies investigating the validity or reliability of radiographs for the functional or structural evaluation of the spine. Of these, 14 were at risk of bias and excluded from the best evidence synthesis [34-44, 47-49]. These included 11 diagnostic studies and eight reliability studies (six of the 11 studies had both diagnostic and reliability components). The diagnostic studies with a risk of bias had methodological limitations including 1 ) inadequate population sampling $(n=5)$, and 2$)$ inadequate blinding $(n=6)$. In the reliability studies with a risk of bias, methodological limitations included: 1) poor population and/or rater sampling $(n=$ $5), 2)$ inadequate inter-rater, intra-rater or information blinding $(n=17), 3)$ no random sampling $(n=4)$ and 4$)$ poor test application and interpretation $(n=3)$ (Tables 1 and 2). We did not identify any cohort, case-control, or cross-sectional studies. Additionally, we did not identify any RCTs, therefore we did not check the clinical trials registry.

We included nine low risk of bias studies in our best evidence synthesis; one diagnostic study [45], seven reliability studies [50-56] and one study with diagnostic and reliability components [46]. One reliability study provided further analyses to previously collected data, which were reported with the original studies [54]. These studies had some methodological limitations, but not in sampling, blinding or random sampling (Tables 1 and 2).

\section{Study characteristics}

We included eight reliability studies, [46, 50-53, 55, 56] five that examined the intra- and inter-rater reliability of Chiropractic BioPhysics ${ }^{\oplus}$, of which four that investigated cervical spine measurements $[51,53,55,56]$ and one 
Table 4 Evidence Tables

\begin{tabular}{|c|c|c|c|c|}
\hline \multicolumn{5}{|c|}{ Reliability Studies } \\
\hline $\begin{array}{l}\text { Author(s), } \\
\text { Year }\end{array}$ & $\begin{array}{l}\text { Design, } \\
\text { Sample size (n) }\end{array}$ & Sample description & Measurement method & Measure of Reliability \\
\hline $\begin{array}{l}\text { Troyanovich, } \\
2000[51]\end{array}$ & $\begin{array}{l}\text { Intra-rater and inter-rater } \\
\text { reliability; } 3 \text { chiropractors } \\
\text { familiar with Chiropractic } \\
\text { BioPhysics }^{\circledast} \text { technique of } \\
\text { measurement } \\
n=36 \text { antero-posterior } \\
\text { cervical spine radiographs }\end{array}$ & $\begin{array}{l}\text { Digitized AP cervical spine } \\
\text { radiographs without artifacts } \\
\text { or other obvious identifying } \\
\text { features with the second } \\
\text { cervical vertebra through } \\
\text { the fourth thoracic vertebra } \\
\text { clearly depicted; from patient } \\
\text { files of a private chiropractic } \\
\text { office }\end{array}$ & $\begin{array}{l}\text { 2-dimensional coordinates of } \\
30 \text { points selected by each } \\
\text { examiner: } R \text { and } L \text { narrow- } \\
\text { waisted-appearing area of } \\
\text { vertebral bodies } T 1-T 4, R \text { and } \\
L \text { narrow-waisted-appearing } \\
\text { area of the articular pillars of } \\
\text { C3-C7, inferolateral aspect of } \\
\text { both superior articular facets } \\
\text { of } C 2 \text {, most superior portion } \\
\text { of spinous process of C2-T4 }\end{array}$ & 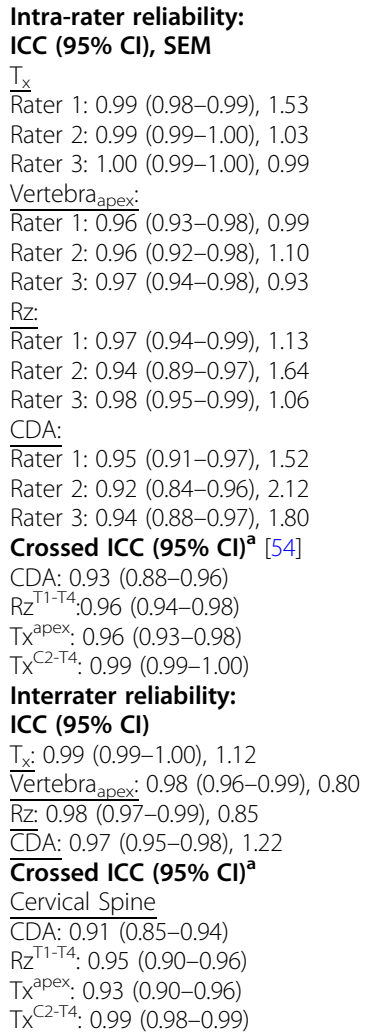 \\
\hline
\end{tabular}

Troyanovich, Intra-rater and Inter-rater 1998 [52]

reliability; 3 chiropractors certified in use of

Chiropractic Biophysics ${ }^{\oplus}$ measurement analysis $n=50$, lateral lumbar radiographs
Lateral lumbar radiographs without artifacts or other identifying features; from patient files of a private chiropractic office
1 rater: $\mathrm{CBP}^{\oplus}$ standard

manual method line drawing of radiographs

2 raters: $\mathrm{CBP}^{\oplus}$ standard

method of analysis using computerized radiographic digitizer

Measurements derived from 17 selected points used to construct following: ARA, ARCU, FERG, COBB, $\mathrm{S}(\mathrm{z}), \mathrm{RRAS}$
Intra-rater reliability

Rater

$\overline{\text { ICC (95 } \% ~ C l) ; ~ S E M) ~}$

T12-L1: $0.54(0.31-0.71) ; 2.16$

L1-L2: $0.75(0.60-0.85) ; 1.82$

L2-L3: $0.77(0.63-0.87) ; 1.44$

L3-L4: $0.85(0.75-0.91): 1.33$

L4-L5: $0.93(0.88-0.96) ; 1.39$

L5-S1: 0.95 (0.92-0.97); 1.68

ARA: 0.97 (0.94-0.98); 1.74

ARCU: 0.99 (0.99-1.00); 0.74

FERG: 0.94 (0.89-0.96); 1.83

COBB: 0.89 (0.81-0.94); 3.07

Sx: $1.00(1.00-1.00) ; 1.07$

Rater 2

$\overline{\text { ICC (95\% CI); SEM) }}$

T12-L1: $0.70(0.53-0.82) ; 1.46$

L1-L2: 0.78 (0.64-0.87); 1.43

L2-L3: $0.61(0.40-0.76) ; 2.30$

L3-L4: 0.66 (0.47-0.79): 2.20

L4-L5: $0.92(0.87-0.95) ; 1.44$

L5-S1: 0.96 (0.94-0.98); 1.49

ARA: 0.98 (0.96-0.99); 1.47

ARCU: 0.93 (0.87-0.96); 2.40

FERG: $0.84(0.73-0.90) ; 2.85$

COBB: 0.88 (0.79-0.93); 3.32

Sx: 0.98 (0.97-0.99); 2.89

Rater 3

$\overline{\text { ICC (95\% CI); SEM) }}$

T12-L1: 0.76 (0.61-0.86); 1.36

L 1-L 2: $0.77(0.63-0.86): 1.48$

L2-L3: $0.71(0.54-0.82) ; 1.73$

L3-L4: $0.70(0.52-0.82) ; 1.77$

L4-L5: 0.91 (0.85-0.95); 1.40 
Table 4 Evidence Tables (Continued)

Reliability Studies

\begin{tabular}{ll}
\hline Author(s), & Design, \\
Year & Sample size (n) \\
\hline
\end{tabular}

Troyanovich, Intra-rater and inter-rater 1995 [53] reliability; 3 chiropractors certified in Chiropractic BioPhysics ${ }^{\circledast}$ method of measurement $n=35$ lateral lumbar radiographs

Sample description

Measurement method

Measure of Reliability

L5-S1: 0.97 (0.95-0.98); 1.40

ARA: 0.96 (0.93-0.98); 1.88

ARCU: 0.87 (0.78-0.92); 3.40

FERG: $0.83(0.73-0.90) ; 2.77$

COBB: $0.95(0.92-0.97) ; 1.99$

Sx: 0.99 (0.98-0.99); 2.14

Inter-rater reliability

Rater 1-2 (manual-computer)

ICC (95\% CI); SEM

ARA L1-5: $0.98(0.96,0.99) ; 1.40$

ARCU: 0.97 (0.95-0.98); 1.48

FERG: $0.88(0.80-0.93) ; 2.42$

COBB: $0.88(0.79-0.93) ; 3.22$

S(z): 0.99 (0.99-1.00); 1.70

RRAs:

T12-L1: 0.68 (0.50-0.81); 1.49

L1-L2: $0.79(0.65-0.87): 1.45$

L2-L3: $0.77(0.63-0.86) ; 1.49$

L3-L4: $0.83(0.71-0.90) ; 1.40$

L4-L5: $0.90(0.84-0.94) ; 1.56$

L5-S1: 0.97 (0.94-9.98); 1.42

Rater 1-3 (manual-computer)

ICC (95\% CI); SEM

ARA L1-5: $0.96(0.93,0.98) ; 1.94$

ARCU: $0.85(0.76,0.91) ; 3.32$

FERG: $0.79(0.65,0.87) ; 3.25$

COBB: $0.83(0.72,0.90) ; 3.78$

S(z): $1.00(0.99,1.00) ; 1.36$

RRAs:

T12-L1: $0.66(0.47,0.79) ; 1.59$

L1-L2: $0.74(0.58,0.84) ; 1.62$

L2-L3: $0.76(0.61,0.85) ; 1.43$

L3-L4: $0.78(0.65,0.87) ; 1.46$

L4-L5: $0.88(0.81,0.93) ; 1.64$

L5-S1: $0.80(0.67,0.88) ; 3.61$

Rater 2-3 (computer-computer)

ICC (95\% CI); SEM

ARA L1-5: $0.96(0.94,0.98) ; 1.76$

ARCU: $0.83(0.73,0.90) ; 3.60$

FERG: $0.84(0.74,0.91) ; 2.63$

COBB: $0.92(0.86,0.95) ; 2.67$

S(z): 0.99 (0.98,0.99); 2.16

RRAs:

T12-L1: $0.63(0.43,0.77) ; 1.57$

L1-L2: $0.72(0.55,0.83) ; 1.53$

L2-L3: $0.72(0.55,0.83) ; 1.67$

L3-L4: $0.72(0.55,0.83) ; 1.70$

L4-L5: $0.90(0.84,0.94) ; 3.50$

L5-S1: 0.81 (0.70,0.89); 3.50

Lateral lumbar radiographs without artifacts or other obvious identifying features selected from patient files of a private, primary-care chiropractic clinic
Arcuate line, Ferguson's sacral-base line, vertical axis line, L1 and L5 stress lines and L 1 and $L 5$ posterior body lines, arcuate angle, relative rotation angle, absolute rotation angle, linear anterior or posterior displacement of the lower thoracic spine
Intra-rater reliability: ICC (95\% CI), SEM

L1-L5

Rater 1: 0.98 (0.92-0.99), 1.48 Rater 2: 0.98 (0.95-0.99), 1.53 Rater 3: 0.98 (0.96-0.99), 1.58 $\mathrm{Sz}$ Rater 1: 0.99 (0.99-1.00), 1.86 Rater 2: 0.97 (0.94-0.98), 4.26 Rater 3: 0.99 (0.98-1.00), 1.97 AA

Rater 1: 0.40 (0.02-0.65), 5.03 Rater 2: 0.81 (0.65-0.90), 2.93

Rater 3: 0.71 (0.49-0.85), 3.53 FERG

Rater 1: 0.97 (0.94-0.98), 1.41

Rater 2: 0.97 (0.94-0.98), 1.45 Rater 3: $0.91(0.82,0.95), 2.12$ L1-L2

$\overline{\text { Rater } 1: 0.87}(0.76,0.93), 1.77$ 
Table 4 Evidence Tables (Continued)

\begin{tabular}{|c|c|c|c|}
\hline \multicolumn{4}{|c|}{ Reliability Studies } \\
\hline $\begin{array}{l}\text { Author(s), } \\
\text { Year }\end{array}$ & $\begin{array}{l}\text { Design, } \\
\text { Sample size (n) }\end{array}$ & Sample description & Measurement method \\
\hline $\begin{array}{l}\text { Haas, } 1990 \\
\text { [50] }\end{array}$ & $\begin{array}{l}\text { Inter-rater reliability; } 2 \\
\text { radiology residents } \\
n=58\end{array}$ & $\begin{array}{l}\text { PA, PA right and left lateral } \\
\text { bending lumbar radiographs of } \\
\text { volunteer students in a } \\
\text { chiropractic institution }\end{array}$ & $\begin{array}{l}\text { Vertebral body rotation and } \\
\text { vertebral body tilting } \\
\text { (intersegmental tilt measurec } \\
\text { as neutral, } L \text { or } R \text { lateral } \\
\text { bending), radiographs } \\
\text { categorized into: } \\
\text { I. Ipsilateral tilt with } \\
\text { contralateral rotation } \\
\text { II. Ipsilateral tilt with } \\
\text { ipsilateral rotation } \\
\text { III. Contralateral tilt with } \\
\text { contralateral rotation } \\
\text { IV. Contralateral tilt with } \\
\text { ipsilateral rotation }\end{array}$ \\
\hline
\end{tabular}

McGregor, Intrarater and interrater reliability; 2 1995 [46] chiropractic radiology residents $n=506$
Neutral lateral, flexion lateral and extension lateral radiographs
Measure of Reliability

Rater 2:0.84 (0.71-0.92), 1.84

Rater 3: 0.94 (0.88-0.97), 1.3

$\mathrm{L} 2-\mathrm{L} 3$

Rater 1: $0.85(0.72-0.92), 1.54$

Rater 2: $0.81(0.66-0.90), 1.31$

Rater 3: $0.80(0.64-0.89), 1.79$

L3-L4

Rater 1: $0.89(0.79-0.94), 1.09$

Rater 2: $0.81(0.66-0.90), 1.52$

Rater 3: $0.78(0.60-0.88), 1.67$

L4-L5

Rater 1: $0.89(0.80-0.94), 1.49$

Rater 2: $0.92(0.85-0.96), 1.17$

Rater 3: 0.87 (0.76-0.93), 1.69

Inter-rater reliability:

ICC (95\%), SEM

L1-L5: 0.98 (0.96-0.99), 1.66

Sz: $0.98(0.97-0.99), 3.20$

AA: $0.66(0.48,0.79), 3.51$

FERG: $0.95(0.91-0.97), 1.73$

L1-L2: $0.88(0.81-0.94), 1.63$

L2-L3: $0.84(0.74-0.91), 1.43$

L3-L4: $0.91(0.85,0.95), 0.97$

L4-L5: 0.93 (0.89-0.96), 1.14

L Lateral Bending Radiograph

Global Motion: K (SE)

$\mathrm{V}=$ overall agreement

Rater 1 and 2

I. $0.63(0.17)$

II. $0.60(0.17)$

III. $0.54(0.17)$

IV. $0.71(0.17)$

V. $0.60(0.10)$

R Lateral Bending Radiograph

Global Motion: K (SE)

$\mathrm{V}=$ overall agreement

Rater 1 and 2

I. $0.64(0.17)$

II. $0.61(0.16)$

III. $0.09(0.17)$

IV. $0.72(0.16)$

V. $0.58(0.10)$

Measure intersegmental motion excursion of each vertebra (\% of sagittal body diameter)
Intrarater reliability:

Generalizability coefficients

C2 Flexion: 0.47

C2 Extension: 0.53

C3 Flexion: 0.66

C3 Extension: 0.68

C4 Flexion: 0.67

C4 Extension: 0.74

C5 Flexion: 0.56

C5 Extension: 0.74

C6 Flexion: 0.65

C6 Extension: 0.59

C7 Flexion: 0.49

C7 Extension 0.07

Interrater reliability:

Generalizability coefficients

C2 Flexion: $0.36-0.43$

C2 Extension: 0.35-0.43

C3 Flexion: 0.60

C3 Extension: 0.67

C4 Flexion: 0.63

C4 Extension: 0.70-0.77

C5 Flexion: $0.55-0.56$

C5 Extension: $0.70-0.71$

C6 Flexion: 0.53-0.58

C6 Extension: $0.50-0.53$

C7 Flexion: 0.02 
Table 4 Evidence Tables (Continued)

\begin{tabular}{|c|c|c|c|}
\hline \multicolumn{4}{|c|}{ Reliability Studies } \\
\hline $\begin{array}{l}\text { Author(s), } \\
\text { Year }\end{array}$ & $\begin{array}{l}\text { Design, } \\
\text { Sample size (n) }\end{array}$ & Sample description & Measurement method \\
\hline $\begin{array}{l}\text { Troyanovich, } \\
1999 \text { [55] }\end{array}$ & $\begin{array}{l}\text { Intra-rater and inter-rater } \\
\text { reliability; } 3 \text { chiropractors } \\
\text { familiar with Chiropractic } \\
\text { BioPhysics }{ }^{\circledR} \text { technique } \\
\text { method of measurement } \\
n=37 \text { anterioposterior I } \\
\text { umbopelvic radiographs }\end{array}$ & $\begin{array}{l}\text { Digitized AP lumbopelvic } \\
\text { radiographs without artifacts } \\
\text { or other obvious identifying } \\
\text { features }\end{array}$ & $\begin{array}{l}\text { 2-dimensional coordinates } \\
\text { of } 33 \text { points selected by each } \\
\text { examiner: } R \text { and } L \text { superior } \\
\text { and inferior corners of each } \\
\text { vertebral body from } T 12 \\
\text { through } L 5 \text {, the most } \\
\text { superior portion of the } \\
\text { spinous processes of T12 } \\
\text { through } L 5 \text { and } S 2 \text {, and the } \\
R \text { and } L \text { superolateral aspects } \\
\text { of the sacral base } \\
\text { Computer calculated lines of } \\
\text { lateral displacement from } \\
\text { true vertical, magnitude of } \\
\text { angle of intersection of two } \\
\text { lines (LDA), angle of } \\
\text { intersection of inferior line } \\
\text { with sacral base line } \\
\text { (LS angle), horizontal line } \\
\text { across sacral base (HB line), } \\
\text { true vertical axis line from } \\
\text { the spinous process of } S 2 \\
\text { cephalically and parallel to } \\
\text { the lateral edge of the } x \text {-ray } \\
\text { film (VAL) }\end{array}$ \\
\hline
\end{tabular}

Jackson, 1993 Intrarater and interrater [56] reliability; 3 chiropractors certified in use of Chiropractic BioPhysics ${ }^{\oplus}$ $n=65$
Lateral cervical films from patient files of a primary care private chiropractic clinic
Measure of Reliability

C7 Extension 0.00

\section{Intra-rater reliability}

ICC $(95 \% \mathrm{CI})$, SEM

$\mathrm{HB}$ angle

Rater 1: 0.72 (0.52-0.84), 1.62

Rater 2: 0.75 (0.57-0.87), 1.78

Rater 3: 0.94 (0.89-0.97), 0.67

LD angle

Rater 1: 0.91 (0.83-0.95), 1.22

Rater 2: 0.90 (0.82-0.95), 1.33

Rater 3: 0.96 (0.92-0.98), 0.87

LS angle

Rater 1: 0.84 (0.72-0.92), 2.04

Rater 2: 0.88 (0.77-0.93), 2.07

Rater 3: 0.96 (0.93-0.98), 0.93

Tx $\frac{T 12}{\text { Rater }}$

Rater 1: 0.97 (0.94-0.98), 1.53

Rater 2: 0.95 (0.91-0.97), 1.95

Rater 3: 0.97 (0.95-0.99), 1.40

Crossed ICC $(95 \% \mathrm{CI})^{\mathrm{a}}$

HB angle: 0.78 (0.67-0.86)

LD angle: 0.92 (0.87-0.95)

LS angle: 0.88 (0.81-0.93)

Tx $x_{T 12-S 1}: 0.96$ (0.94-0.98)

Inter-rater reliability

ICC $(\mathbf{9 5} \% \mathrm{CI})$, SEM

HB angle: 0.71 (0.56-0.82), 1.62

LD angle: 0.97 (0.94-0.98), 0.75

LS angle: 0.83 (0.73-0.90), 2.13

$\mathrm{Tx}^{\mathrm{T} 12}$ : 0.95 (0.91-0.97), 2.01

Crossed ICC $(95 \% \mathrm{Cl})^{\mathrm{a}}[54]$

HB angle: 0.61 (0.49-0.73)

LD angle: $0.89(0.83-0.94)$

LS angle: $0.76(0.66-0.85)$

$\mathrm{TX}_{\mathrm{T} 12-\mathrm{S} 1}$ : 0.92 (0.88-0.95)

Standard $\mathrm{CBP}^{\oplus}$ measurement Intra-rater reliability protocols: Atlas plane line, Ruth Jackson's stress lines, vertical axis line and C2 through C7 posterior body lines; relative rotation angle measurements, ARA
Not reported due to inadequate statistics used to compute reliability. Inter-rater reliability

Bartko's ICC; SEM

Atlas plane line:

0.93; 1

ARA:

0.96; $1.20^{\circ}$

Anterior head translation:

$0.80 ; 1.23 \mathrm{~mm}$ Intersegmental angle C2-3:

$0.72 ; 0.57^{\circ}$

Intersegmental angle C3-4:

$0.79 ; 0.54^{\circ}$

Intersegmental angle C4-5:

$0.86 ; 1.04^{\circ}$

Intersegmental angle C5-6:

$0.79 ; 0.66^{\circ}$

Intersegmental angle C6-7:

$0.74 ; 0.65^{\circ}$

$A A$ arcuate angle; $A R A$ absolute rotation angle; $A R C U$ arcuate angle measurement; $C B P^{\oplus}$ Chiropractic Biophysics ${ }^{\oplus}$; $C D A$ cervicodorsal angle; $C O B B$ Cobb angle measurement; FERG Ferguson's angle measurement; $H B$ angle angle of sacral base compared to horizontal; $H B$ line horizontal line intersection line drawn across the sacral base; $L$ left; $L D$ angle lumbo-dorsal angle, angle of best fit line form lumbar apex to $L 5$ compared to the sacral base; $L S$ angle lumbosacral angle, angle of best fit line from lumbar apex to L5 compared to the sacral base; $R$ right; $R R A$ intersegmental measurements; $R y$ segmental axial rotation angles; $R z$ magnitude of the angle of intersection between vertical axis line and lower most line; $S E M$ standard error of measurement; $S(z)$ translation measurement of lower thoracic spine to $S 1 ; T_{x}$ perpendicular distance from vertical axis line to the center of the vertebral body of $C 2 ; T_{X}^{T 12}$ lateral translation distance of T12 compared to 9S2; VAL vertical axis line; vertebra apex: linear distance from center of vertebra most displaced from line connecting the centers of $\mathrm{C} 2$ and $\mathrm{T} 4$

${ }^{a}$ Harrison 2002 [54] calculated crossed ICCs from two individual studies, these calculations are presented with the original articles 


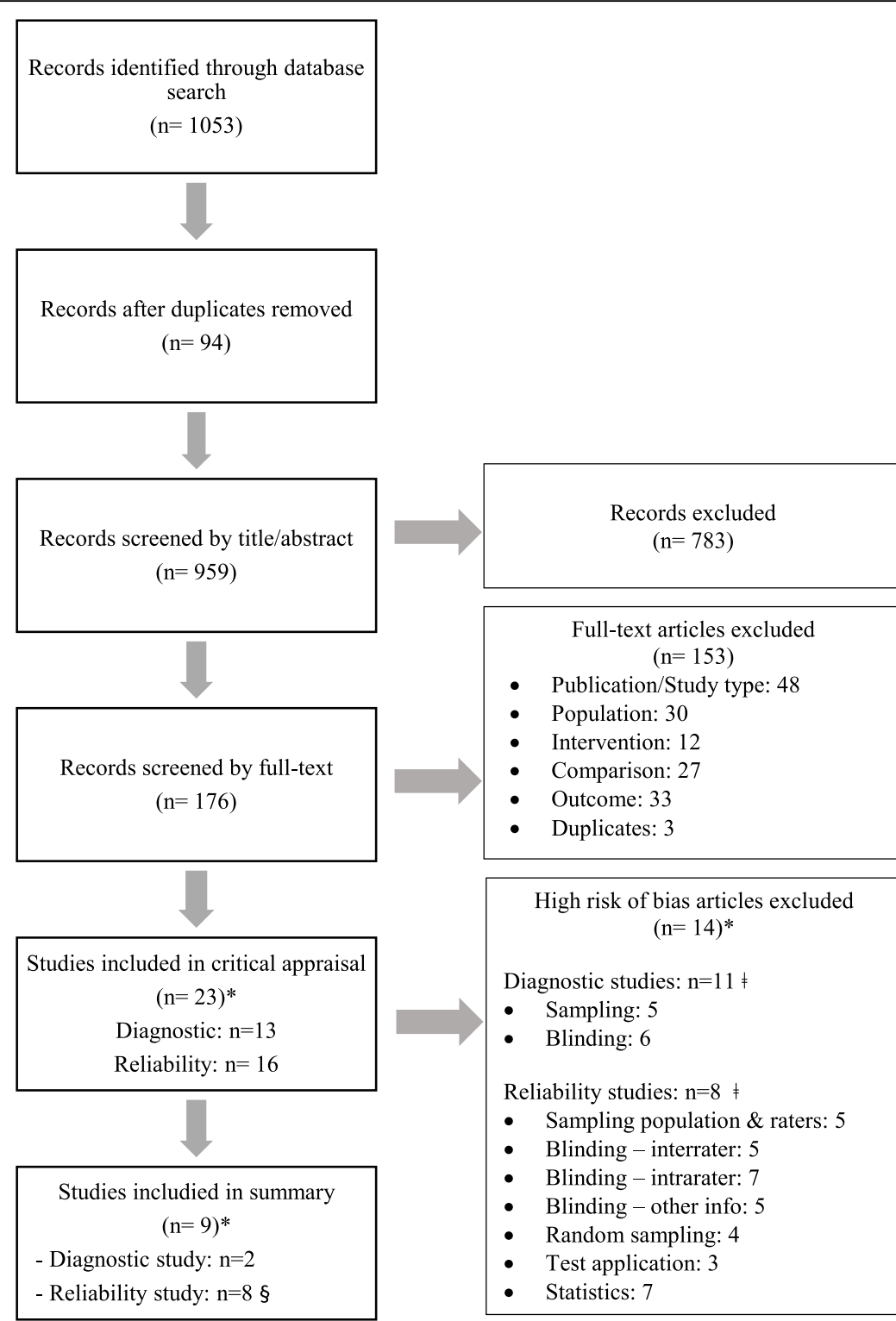

*Some records had diagnostic and reliability components

\# Studies may have more than one criterion that led to exclusion

$\S$ Harrison, 2002 reported additional calculations of previously collected data, presented with original studies

Fig. 2 Flow diagram of study selection

that studied lumbar spine measurements [52]. One study examined the intra- and inter-rater reliability of flexionextension radiographs in addition to a standard cervical radiograph series, [46] and one investigated the interrater reliability of vertebral rotation and tilt of lateral bending radiographs [50]. We included two phase two diagnostic (validity) studies, $[45,46]$ that investigated whether patients with radiographic findings were more likely to have the target disorder than patients with other test outcomes [28]. One study investigated radiographic findings of spinal degeneration and cervical complaints [45] and the other investigated findings on flexion-extension radiographs of intersegmental clinical hypermobility [46].

\section{Reliability of radiographic measurements}

Four studies investigating Chiropractic BioPhysics ${ }^{\bullet}$ measurements of the cervical spine (i.e., anterior head translation, vertebral translation in the cervical and thoracic spine, cervical lordosis angle, cervicodorsal angle, absolute rotation angle, Ferguson's angle, Cobb angle and intersegmental measurements) found that these were 
performed with acceptable levels of reliability (Tables 3 and 4) $[51,53,55,56]$. One study investigated Chiropractic BioPhysics ${ }^{\circ}$ measurements of the lumbar spine (i.e., sacral base angle, lumbodorsal angle, lumbosacral angle and lumbar spine vertebral translation) also reported acceptable levels of reliability [52]. The one exception was the measurement of the arcuate angle, which had a low to acceptable level of reliability in the cervical and lumbar spine [52, 53].

For other radiographic measurements, Haas et al. found that categorizing vertebral body rotation and tilting into five categories, may be associated with poor reliability and significant measurement error [50]. Similarly, McGregor et al. reported that measuring intersegmental motion of each vertebra in flexion and extension is associated with poor reliability and significant measurement error [46].

\section{Validity of radiographic measurements}

We did not identify any studies of acceptable methodological quality providing evidence of the diagnostic accuracy (sensitivity, specificity, predictive values) of Chiropractic BioPhysics ${ }^{\circ}$ measurements. Thus, we do not know if these measurements are evaluating clinically important outcomes for conditions of the cervical or lumbar spine.

Two low risk of bias studies provided preliminary evidence, phase two diagnostic studies, of the diagnostic validity of using radiographs for functional and structural evaluation of the spine $[45,46]$. McAviney et al. [45] investigated the association of cervical radiograph measurements in patients with and without cervical spine complaints. The authors did not find significant differences in head anterior weight bearing between participants with or without cervical complaints [45]. However, they reported that participants with less than $20^{\circ}$ of absolute rotation angle (a measure of cervical lordosis) were greater than two times more likely to have cervical complaints compared to those who had more than $20^{\circ}$ [45]. McGregor et al. [46] investigated the benefit of adding cervical flexion-extension radiographs to a normal series of cervical radiographs and standardized case report for the diagnosis of intersegmental clinical hypermobility. They reported no additional diagnostic benefit of using flexion-extension radiographs [46].

\section{Clinical utility}

We did not identify any relevant studies investigating the diagnostic or therapeutic utility of cervical, thoracic or lumbar radiographs (in the absence of red flags) for the functional or structural evaluation of the spine. Similarly, we did not identify any relevant studies that investigated whether functional or structural findings on repeat radiographs of the cervical, thoracic or lumbar spine are valid markers of clinically meaningful change when monitoring conditions or managing patients.

\section{Discussion}

Clinical utility refers to the degree to which the use of a test (such as radiographs) is associated with changing health outcomes through diagnosis or selection of an appropriate treatment [17-19]. We did not find evidence that cervical, thoracic or lumbar radiographs (in the absence of red flags) obtained for the purpose of evaluating the function or structure evaluation of the spine can benefit patients. Therefore, we do not recommend that routine, or repeat radiographs of the cervical, thoracic or lumbar spine (in the absence of red flags) be used by chiropractors to evaluate the structure or function of the spine for diagnostic or therapeutic purposes.

Although we found eight reliability studies and two diagnostic (phase two) validity studies with a low risk of bias, these studies cannot be used to justify using routine or repeat radiographs of the spine [45, 46, 50-56]. While some measurements of cervical and lumbar spine radiographs have acceptable levels of reliability, and preliminary evidence of diagnostic validity, we did not identify any acceptable studies investigating their clinical utility.

Several evidence-based clinical practice guidelines are available to inform the use of radiographs in cases of trauma, or when pathology is suspected [3-5, 7]. Moreover, guidelines make clear recommendations against the use of radiographs to assess function of the spine [5, 7]. While our rapid review agrees with these statements, it nevertheless conflicts with recommendations published by the International Chiropractic Association in the document entitled: 'Practicing Chiropractors' Committee on Radiology Protocols (PCCRP) for Biomechanical Assessment of Spinal Subluxation in Chiropractic Clinical Practice", a guideline frequently referenced by a subset of chiropractors [13]. The divergent conclusions are attributable to differences in methodology, in particular differences in the search strategy and selection of articles. The development of the PCCRP document did not include a risk of bias assessment of eligible studies. Thus the synthesis included low quality studies which likely biased the recommendations made by that guideline expert panel [59]. Furthermore, it is unclear whether the guideline expert panel had editorial independence; most members $(17 / 25)$ of the guideline expert panel and investigators were members of the sponsoring organization [13].

In a review by Triano et al. [60], they used a consensus process to assess the appropriateness of imaging as a diagnostic tool to guide the use of manual therapy. Despite the low quality and narrative nature of their review, the use of radiographs to localize the site of care for manual therapy was not recommended. However, contrary to our findings, 
they recommended the use of static and motion radiographic studies to identify hypermobile but not hypomobile segments. Our study included two relevant low risk of bias studies $[46,50]$ suggesting poor reliability of radiographs to assess motion patterns, and one preliminary phase two diagnostic study [46] that was not included in their review, that clearly contradicts their recommendation.

We live in the era of value-based health care [61]. One of the goals of value-based healthcare is to reduce the utilization of low-value tests and interventions that do not benefit patients but increase the costs of care. Campaigns such as Choosing Wisely have been designed and implemented to promote conversations between clinicians and patients by helping patients choose care that is: 1) supported by evidence; 2) not duplicative of other tests or procedures already received; 3 ) free from harm; and 4) truly necessary [62]. In 2017, the American Chiropractic Association adapted the Choosing Wisely ${ }^{\circ}$ recommendations on lumbar spine radiography [63] and recommended to avoid routine spinal imaging in the absence of clear clinical indicators for patients with acute low back pain of less than 6 weeks duration. Furthermore, the American Chiropractic Association recommended that repeat imaging must not be used to monitor patients' progress [62]. Our findings are in agreement with the American Chiropractic Association adapted Choosing Wisely recommendations.

A principle of value-based health care is that clinical interventions should be free from harm, or at the very least, the benefits of the intervention must substantially outweigh the risks [63]. A known risk for ionizing exposure is the increased frequency of cancer beyond that occurring spontaneously and non-cancer diseases (i.e. cardiovascular diseases) [64-66]. Studies have shown that $100 \mathrm{mSv}$ is the approximate dose of radiation to be received by a patient before there is a known increased risk of cancer over a lifetime $[64,67,68]$. The current widely used theory on radiation accumulation is based on the linear no-threshold (LNT) model which in simple terms states: no dose of radiation exists without risk and that risk increases proportionally with dose $[68,69]$. Currently, direct risks associated with low doses, as those received with radiographic studies, in the LNT model are unknown [64-66].

However, despite the ongoing debate of the LNT theory, $[70,71]$ the argument remains that radiographic studies should not be considered in isolation, but viewed as part of the patient's lifetime exposure. Ionizing radiation is a cumulative process that occurs from natural sources, such as sunlight, and decay of elements in our environment, as well as man-made sources, such as medical imaging (i.e. radiographs, computed tomography (CT) and nuclear medicine scans) [62,63]. It is therefore recommended by the International Commission on
Radiological Protection (ICRP) and the Canadian $\mathrm{Nu}$ clear Safety Commission (CNSC), that in the absence of information pertaining to low-dose risks, to follow the "as low as reasonably achievable" (ALARA) principle [64]. ALARA is not a dose limit, but a practice that aims to keep the dose levels as far as possible below the regulatory limit $[64,72]$. In light of the inherent risks of the use of ionizing radiation, and given that the clinical utility is unknown, the use of routine and repeat radiographs for the purpose of assessing functional or structural evaluation of the spine is not recommended.

Our rapid review has limitations inherent to the rapid review methodology [21]. These limitations include: 1) focused search of the literature (three databases) which may lead to studies being omitted from the review; and 2) the conduct of screening, critical appraisal and data extraction done by one investigator instead of two. However, we reduced the impact of these limitations by: carefully selecting databases where the relevant literature is most likely to be published (MEDLINE, CINAHL, and ICL); and implementing a structured quality assurance methodology to minimize error in screening and selection of articles, and data extraction.

\section{Conclusion}

Radiographs are an important diagnostic tool in patient management when clinical indicators of serious pathologies (red flags) are present. We found no evidence that radiographs used to assess the function or structure of the spine improves patients' outcomes. Therefore, in the absence of red flags, and given the inherent risks of ionizing radiation, we do not recommend the clinical use of radiographs for the routine and repeat evaluation of the structure and function of the spine.

\section{Supplementary information}

Supplementary information accompanies this paper at https://doi.org/10. 1186/s12998-020-00323-8.

Additional file 1. MEDLINE Search Strategy.

Additional file 2. Glossary.

\section{Abbreviations \\ ALARA: As low as reasonably achievable; CNSC: Canadian Nuclear Safety Commission; ICRP: International Commission on Radiological Protection; $\mathrm{RCT}$ : Randomized controlled trials}

\section{Acknowledgements}

This study was supported by the College of Chiropractors of British Columbia to Ontario Tech University. The College of Chiropractors of British Columbia was not involved in the design, conduct or interpretation of the research that informed the research. This research was undertaken, in part, thanks to funding from the Canada Research Chairs program to Pierre Côté who holds the Canada Research Chair in Disability Prevention and Rehabilitation at Ontario Tech University, and from the Canadian Chiropractic Research Foundation to Carol Cancelliere who holds a Research Chair in Knowledge Translation in the Faculty of Health Sciences at Ontario Tech University. We 
would also like to acknowledge Anne Taylor-Vaisey, BA, MLS, who designed and conducted the systematic search of the literature.

\begin{abstract}
Authors' contributions
MC assisted in developing research questions and rapid review methodology, was the main reviewer for article screening, critical appraisal and data extraction, drafted the manuscript, and reviewed and revised the manuscript. CC assisted in developing research questions and rapid review methodology, was the second reviewer for inter-rater reliability of screening phase one, verified critical appraisal and data extraction, and co-drafted and reviewed the manuscript. PC, SM, VK and AS assisted in developing research questions and rapid review methodology, verified critical appraisal and data extraction, and reviewed and revised the manuscript. All authors approved the final manuscript as submitted and agree to be accountable for all aspects of the work.
\end{abstract}

\section{Funding}

This study was supported by the College of Chiropractors of British Columbia to Ontario Tech University. The College of Chiropractors of British Columbia was not involved in the design, conduct or interpretation of the research that informed the research. This research was undertaken, in part, thanks to funding from the Canada Research Chairs program to Pierre Côté who holds the Canada Research Chair in Disability Prevention and Rehabilitation at Ontario Tech University, and funding from the Canadian Chiropractic Research Foundation to Carol Cancelliere who holds a Research Chair in Knowledge Translation in the Faculty of Health Sciences at Ontario Tech University.

\section{Availability of data and materials}

The datasets used and/or analyzed during the current study are available from the corresponding author on reasonable request.

\section{Ethics approval and consent to participate}

Not applicable.

\section{Consent for publication}

Not applicable.

\section{Competing interests}

Pierre Côté reports research grants unrelated to this work from Aviva Canada, Canadian Institutes of Health Research—Canada Research Chair Program, French Chiropractic Association, Ontario Ministry of Finance, Ontario Trillium Foundation; travel expenditures unrelated to this work from Griffith University - Whiplash Symposium 2017, Southern Denmark University, Institut Franco-Europeen de Chiropraxie, Karolinska Institutet, North American Spine Society, University of Quebec-Trois-Rivieres, University of Zurich, World Federation of Chiropractic; fees medical-legal expertise from the Canadian Chiropractic Protective Association. Carol Cancelliere reports a research grant unrelated to this work from the Canadian Chiropractic Research Foundation - Research Chair in Knowledge Translation in the Faculty of Health Sciences, Ontario Tech University, Canada. Silvano Mior reports research grants unrelated to this work from the Canadian Chiropractic Association, Ontario Chiropractic Association and Ontario Ministry to Seniors Affairs; travel expenditures unrelated to this work from Institut Franco-Europeen de Chiropraxie, World Federation of Chiropractic and University of Norway.

\section{Author details}

${ }^{1}$ Faculty of Health Sciences, Ontario Tech University and Centre for Disability Prevention and Rehabilitation, 2000 Simcoe St N, Oshawa, ON L1G 0C5, Canada. ${ }^{2}$ Canadian Memorial Chiropractic College, North York, Ontario, Canada.

\section{Received: 31 March 2020 Accepted: 24 May 2020} Published online: 09 July 2020

\section{References}

1. Bussières $A E$, Sales $A E$, Ramsay $T$, Hilles $S$, Grimshaw JM. Practice patterns in spine radiograph utilization among doctors of chiropractic enrolled in a provider network offering complementary care in the United States. J Manip Physiol Ther. 2013;36:127-42. https://doi.org/10.1016/j.jmpt.2013.04.002.
2. Mizrahi D, Parker L, Zoga A, Levin D. National Trends in the utilization of skeletal radiography from 2003 to 2015. J Am Coll Radiol. 2018;15:1408-14.

3. Côté P, Yu H, Shearer HM, Randhawa K, Wong JJ, Mior S, et al. Nonpharmacological management of persistent headaches associated with neck pain: A clinical practice guideline from the Ontario protocol for traffic injury management (OPTIMa) collaboration. Eur J Pain (United Kingdom). 2019;January:1051-70.

4. Côté P, Wong JJ, Sutton D, Shearer HM, Mior S, Randhawa K, et al. Management of neck pain and associated disorders: a clinical practice guideline from the Ontario protocol for traffic injury management (OPTIMa) collaboration. Eur Spine J. 2016:25:2000-22.

5. Bussières AE, Taylor JAM, Peterson C. Diagnostic imaging practice guidelines for musculoskeletal complaints in adults-an evidence-based approach-part 3: spinal disorders. J Manip Physiol Ther. 2008;31:33-88.

6. Patel N, Broderick D, Burns J, Deshmukh T, Fries I, Harvey H, et al. ACR appropriateness criteria ${ }^{\oplus}$ low Back pain. J Am Coll Radiol. 2016;13:1069-78.

7. Stiell I, Clement C, McKnight R, Brison R, Schull M, Rowe B, et al. The Canadian C-spine rules versus the NEXUS low-risk criteria in patients with trauma. N Engl J Med. 2003:349:2510-8.

8. Henschke N, Maher C, Ostelo R, de Vet H, Macaskill P, Irwig L. Red flags to screen for malignancy in patients with low-back pain. Cochrane Database Syst Rev. 2013;28:CD008686.

9. Hawk C, Schneider MJ, Haas M, Katz P, Dougherty P, Gleberzon B, et al. Best practices for chiropractic Care for Older Adults: a systematic review and consensus update. J Manip Physiol Ther. 2017:40:217-29. https://doi.org/10. 1016/j.jmpt.2017.02.001.

10. Amorin-Woods LG, Parkin-Smith GF. Clinical decision-making to facilitate appropriate patient management in chiropractic practice: "The 3-questions model.". Chiropr Man Ther. 2012;20:1-6

11. Messer L, Sibilia J, Miazhiom ACL. Diagnostic uncertainty and clinical decision-making strategies. Jt Bone Spine. 2018;85:267-9. https://doi.org/10. 1016/j.jbspin.2017.12.006.

12. Jenkins HJ, Downie AS, Moore CS, French SD. Current evidence for spinal Xray use in the chiropractic profession: a narrative review. Chiropr Man Ther. 2018;26:1-11.

13. Harrison D, Harrison D, Kent C, Betz J. Practicing Chiropractors' Committee on Radiology Protocols (PCCRP) For Biomechanical Assessment of Spinal Subluxation in Chiropractic Clinical Practice; 2009. p. 1-387.

14. Harrison DD, Siskin LA. ICA Best Practices \& Practice Guidelines Chapter 6. In: Routine Plain Film Radiography is the Standard of Practice in Chiropractic: 2008

15. Coleman RR, Wolf HK, Lopes AM, Coleman MJ. History or science: the controversy over chiropractic Spinography. Chiropr Hist. 2013;33(I):66-81 http://ezproxy.cqu.edu.au/login?url=http://search.ebscohost.com/login. aspx?direct=true $\& \mathrm{db}=c 8 \mathrm{~h} \& \mathrm{AN}=2012162044 \&$ site=eds-live\&scope $=$ site

16. Young KJ. Evaluation of publicly available documents to trace chiropractic technique systems that advocate radiography for subluxation analysis: a proposed genealogy. J Chiropr Humanit. 2014;21:1-24. https://doi.org/10. 1016/j.echu.2014.09.001.

17. Lesko $\sqcup$, Zineh I, Huang SM. Editorial: what is clinical utility and why should we care? Clin Pharmacol Ther. 2010;88:729-33. https://doi.org/10.1038/clpt. 2010.229

18. Bossuyt PMM, Reitsma JB, Linnet K, Moons KGM. Beyond diagnostic accuracy: the clinical utility of diagnostic tests. Clin Chem. 2012;58:1636-43.

19. Nelson-Gray RO. Treatment utility of psychological assessment. Psychol Assess. 2003;15:521-31.

20. About the College of Chiropractors of BC. 2020. https://www.chirobc.com/ about-the-college-of-chiropractors-of-bc/. Accessed 12 Mar 2020.

21. Tricco AC, Langlois EV, Straus SE, editors. Rapid reviews to strengthen health policy and systems: a practical guide. Geneva: World Health Organization; 2017.

22. Tricco AC, Antony J, Zarin W, Strifler L, Ghassemi M, Ivory J, et al. A scoping review of rapid review methods. BMC Med. 2015. https://doi.org/10.1186/ s12916-015-0465-6.

23. Corso M, Cancelliere C, Mior S, Taylor-vaisey A, Côté P. The safety of spinal manipulative therapy in children under 10 years: a rapid review; 2020. p. 1-18.

24. Moher D, Liberati A, Tetzlaff J, Altman DG, Group P. Preferred reporting items for systematic reviews and meta-analyses: the PRISMA statement. PLOS Med. 2009:6:e1000097.

25. Zorzela L, Loke YK, loannidis JP, Golder S, Santaguida P, Altman DG, et al. PRISMA harms checklist: improving harms reporting in systematic reviews. BMJ. 2016;352:1157. 
26. Fletcher $\mathrm{RH}$, Fletcher SW, Wagner EH. Clinical epidemiology the essentials. Third edit. Baltimore, Maryland: Williams \& Wilkins; 1996.

27. Korevaar DA, Gopalakrishna G, Cohen JF, Bossuyt PM. Targeted test evaluation: a framework for designing diagnostic accuracy studies with clear study hypotheses. Diagnostic Progn Res. 2019;3:1-10.

28. Sackett D, Haynes R. Evidence base of clinical diagnosis. The artchitecture of diagnostic research. Br Med J. 2002;324:602-6.

29. Harbour R, Miller K. A new system for grading recommendations in evidence based guidelines. Br Med J (Clinical Res Ed). 2001;323:334-6.

30. Scottish Intercollegiate Guidelines Network Critical Appraisal: Notes and checklists. 2013. http://www.sign.ac.uk/methodology/checklists.html\#.

31. Hoy D, Brooks P, Woolf A, Blyth F, March L, Bain C, et al. Assessing risk of bias in prevalence studies: modification of an existing tool and evidence of interrater agreement. J Clin Epidemiol. 2012;65:934-9. https://doi.org/10. 1016/j.jclinepi.2011.11.014.

32. Whiting P, Weswood M, Rutjes A, Reitsma J, Bossuyt P, Kleijnen J. Evaluation of QUADAS, a tool for the quality assessment of diagnostic accuracy studies. BMC Med Res Methodol. 2006;6:9.

33. Lucas $N$, Macaskill $P$, Irwig L, Moran R, Rlckards L, Turner R, et al. The reliability of a quality appraisal tool for studies of diagnostic reliability (QAREL). BMC Med Res Methodol. 2013;9:111.

34. Gregory P, Hayek R, Mann-Hayek A. Correlating motion palpation with functional $x$-ray findings in patients with low back pain. Australas Chiropr Osteopat. 1998;7:15-159.

35. Harrison D, Cailliet R, Harrison DD, Janik T, Holland B. Changes in sagittal lumbar configuration with a new method of extension traction: nonrandomized clinical controlled trial. Arch Phys Med Rehabil. 2002;83: 1585-91.

36. Frymoyer JW, Phillips RB, Newberg AH, MacPherson BV. A comparative analysis of the interpretations of lumbar spinal radiographs by chiropractors and medical doctors. Spine (Phila Pa 1976). 1986;11:1020-3.

37. Harrison DE, Harrison DD, Betz JJ, Janik TJ, Holland B, Colloca CJ, et al. Increasing the cervical lordosis with chiropractic biophysics seated combined extension-compression and transverse load cervical traction with cervical manipulation: nonrandomized clinical control trial. J Manip Physiol Ther. 2003:26:139-51.

38. Wight S, Osborne N, Breen AC. Incidence of ponticulus posterior of the atlas in migraine and cervicogenic headache. J Manip Physiol Ther. 1999;22:15-20.

39. Rosok G, Peterson CK. Comparison of the sacral base angle in females with and without spondylolysis. J Manip Physiol Ther. 1993;16:447-52.

40. Haas M, Peterson D. A roentgenological evaluation of the relationship between segmental motion and malalignment in lateral bending. J Manip Physiol Ther. 1992;15:350-60.

41. Haas M, Nyiendo J. Lumbar motion trends and correlation with low back pain. Part II. A roentgenological evaluation of quantitative segmental motion in lateral bending. J Manip Physiol Ther. 1992;15:224-34.

42. Leboeuf C, Kimber D, White K. Prevalence of spondylolisthesis, transitional anomalies and low intercrestal line in a chiropractic patient population. J Manip Physiol Ther. 1989;12:200-4.

43. Phillips RB, Frymoyer JW, MacPherson BV, Newburg AH. Low back pain: a radiographic enigma. J Manip Physiol Ther. 1986;9:183-7.

44. Rudy SS, Poulos A, Owen L, Batters A, Kieliszek K, Willox J, et al. The correlation of radiographic findings and patient symptomatology in cervical degenerative joint disease: a cross-sectional study. Chiropr Man Ther. 2015;23:1-7.

45. McAviney J, Schulz D, Bock R, Harrison DE, Holland B. Determining the relationship between cervical lordosis and neck complaints. J Manip Physiol Ther. 2005;28:187-93.

46. McGregor M, Mior S, Shannon H, Hagino C, Schut B. The clinical usefulness of flexion-extension radiographs in the cervical spine. Top Clin Chiropr. 1995;2:19-83.

47. Assendelft WJJ, Bouter LM, Knipschild PG, Wilmink JT. Reliability of lumbar spine radiograph reading by chiropractors. Spine. 1997;22:1235-41.

48. Plaugher $\mathrm{G}$, Cremata EE, Phillips RB. A retrospective consecutive case analysis of pretreatment and comparative static radiological parameters following chiropractic adjustments. J Manip Physiol Ther. 1990;13:498-506.

49. Janik T, Harrison DE, Harrison DD, Holland B, Coleman RR, Payne MR. Reliability of lateral bending and axial rotation with validity of a new method to determine axial rotation on anteroposterior cervical radiographs. J Manip Physiol Ther. 2001;24:445-8.

50. Haas M, Nyiendo J, Peterson C, Thiel H, Sellers T, Cassidy D, et al. Interrater reliability of roentgenological evaluation of the lumbar spine in lateral bending. J Manip Physiol Ther. 1990;13:179-89.
51. Troyanovich SJ, Harrison D, Harrison DD, Harrison SO, Janik T, Holland B. Chiropractic biophysics digitized radiographic mensuration analysis of the anteroposterior cervicothoracic view: a reliability study. J Manip Physiol Ther. 2000;23:476-82.

52. Troyanovich SJ, Harrison DE, Harrison DD, Holland B, Janik TJ. Further analysis of the reliability of the posterior tangent lateral lumbar radiographic mensuration procedure: concurrent validity of computer- aided X-ray digitization. J Manip Physiol Ther. 1998;21:460-7.

53. Troyanovich SJ, Robertson GA, Harrison DD, Holland B. Intra- and interexaminer reliability of the chiropractic biophysics lateral lumbar radiographic mensuration procedure. J Manip Physiol Ther. 1995;18:519-24.

54. Harrison DE, Holland B, Harrison DD, Janik TJ. Further reliability analysis of the Harrison radiographic line-drawing methods: crossed ICCs for lateral posterior tangents and modified Risser-Ferguson method on AP views. J Manip Physiol Ther. 2002;25:93-8.

55. Troyanovich S, Harrison S, Harrison D, Harrison D, Payne M, Janik T, et al. Chiropractic biophysics digitized radiographic mensuration analysis of the anteroposterior lumbopelvic view: a reliability study. J Manip Physiol Ther. 1999;22:476-82

56. Jackson BL, Harrison DD, Robertson GA, Barker WF. Chiropractic biophysics lateral cervical film analysis reliability. J Manip Physiol Ther. 1993;16:384-91.

57. Slavin R. Best evidence synthesis: an intelligent alternative to meta-analysis. J Clin Epidemiol. 1995;48:9-18.

58. Campbell M, McKenzie JE, Sowden A, Katikireddi SV, Brennan SE, Ellis S, et al. Synthesis without meta-analysis (SWiM) in systematic reviews: reporting guideline. BMJ. 2020;368:16890.

59. Roberts I, Ker K. How systematic reviews cause research waste. Lancet. 2015; 386:1536.

60. Triano JJ, Budgell B, Bagnulo A, Roffey B, Bergmann T, Cooperstein R, et al. Review of methods used by chiropractors to determine the site for applying manipulation. Chiropr Man Ther. 2013;21:1. https://doi.org/10.1186/ 2045-709X-21-36.

61. What Is Value-Based Healthcare? NEJM Catal. 2017. https://doi.org/10.1056/ CAT.17.0558.

62. Imaging Tests for Low Back Pain: When you need them — and when you don't. 2017. https://choosingwiselycanada.org/wp-content/uploads/2017/05/ Low-Back-Pain-EN.pdf.

63. American Chiropractic Association Five Things Physicians and Patients Should Question. Choosing Wisely. 2019. https://www.choosingwisely.org/ societies/american-chiropractic-association/. Accessed 16 Mar 2020.

64. Introduction To Radiation. Canadian Nuclear Safety Commission. 2012. https://nuclearsafety.gc.ca/eng/pdfs/Reading-Room/radiation/Introductionto-Radiation-eng.pdf.

65. Sodickson A, Baeyens P, Andriole K, Prevedello L, Nawfel R, Hanson R, et al. Recurrent $C T$, cumulative radiation exposure, and associated radiationinduced Cancer risks from CT of adults. Radiology. 2009;251:175-84.

66. Kamiya K, Ozasa K, Akiba S, Niwa O, Kodama K, Takamura N, et al. Longterm effects of radiation exposure on health. Lancet. 2015;386:469-78. https://doi.org/10.1016/S0140-6736(15)61167-9.

67. Mettler FA, Huda W, Yoshizumi TT, Mahesh M. Effective doses in radiology and diagnostic nuclear medicine: a catalog. Radiology. 2008;248:254-63.

68. Lin EC. Radiation risk from medical imaging. Mayo Clin Proc. 2010;85:1142-6.

69. Linear-Non-Threshold Model. Canadian Nuclear Safety Commission; government of Canada. 2015. https://nuclearsafety.gc.ca/eng/resources/ health/linear-non-threshold-model/index.cfm. Accessed 13 Mar 2020.

70. Kawchuk G, Goertz C, Axén I, Descarreaux M, French S, Haas M, et al. Letter to the Editor Re: Oakley PA, Cuttler JM, Harrison DE. X-Ray Imaging Is Essential for Contemporary Chiropractic and Manual Therapy Spinal Rehabilitation: Radiography Increases Benefits and Reduces Risks. Dose Response. 2018 Jun 19;16(2). Dose-Response. 2018;16:1-2.

71. Bussières $A E$, Ammendolia $C$, Peterson C, Taylor JAM. Ionizing radiation exposure--more good than harm? The preponderance of evidence does not support abandoning current standards and regulations. J Can Chiropr Assoc. 2006;50:103-6.

72. Uffmann M, Schaefer-Prokop C. Digital radiography: the balance between image quality and required radiation dose. Eur J Radiol. 2009;72:202-8.

\section{Publisher's Note}

Springer Nature remains neutral with regard to jurisdictional claims in published maps and institutional affiliations. 